\title{
Study on Dual-Concentric-Core Dispersion Compensating Photonic Crystal Fiber
}

\author{
Md. Selim Habib, Md. Samiul Habib, S.M.A. Razzak \\ Department of Electrical \& Electronic Engineering, Rajshahi University of \\ Engineering \& Technology, Rajshahi-6204, Bangladesh \\ E-mail: selim041073@yahoo.com
}

\begin{abstract}
The dual-concentric-core photonic crystal fiber composed of pure silica and air is proposed in this paper. Around $1.55 \mu \mathrm{m}$, it exhibits a negative chromatic dispersion as high as $-20000 \mathrm{ps} /(\mathrm{km} . \mathrm{nm})$. The finite element method with perfectly matched absorbing layers boundary condition is used to investigate the guiding properties. The explanations to propagation states of the fundamental mode and the second mode are given elaborately
\end{abstract}

Keywords: Photonic crystal fiber, chromatic dispersion, finite element method.

\section{Introduction}

Photonic crystal fibers (PCFs) or holey fibers or microstructure optical fibers [1] have a microscopic array of air channels running down their length that make a low index cladding around the undoped silica core. Such holey claddings in PCFs help tuning dispersion slope and controlling confinement losses in a way that is not possible in conventional fibers [2]. PCFs technology has rapidly progressed in recent years and attracted much attention for fiber device applications because of its superior optical properties [3]-[7]; for example, very high or very low nonlinearity, wideband dispersion-flattened characteristics, high birefringence, endlessly single mode guiding, high negative dispersion, and many others.

Chromatic dispersion is one of the most fundamental characteristics of optical fiber. The pulse is broadened mostly because of this factor. At present, millions of kilometers of installed optical links around the world operate with $1.31 \mu \mathrm{m}$ 
optimized G.652 type of single-mode fibers. Due to the availability of the erbiumdoped fiber amplifiers (EDFA) and also because of lower loss at the $1.55 \mu \mathrm{m}$ band, there has been a substantial interest to operate these installed fibers at the $1.55 \mu \mathrm{m}$ band. Unfortunately, when operated at the $1.55 \mu \mathrm{m}$ band these fibers exhibit chromatic dispersion of 10-17 ps/(km.nm). In a long-haul optical link, the chromatic dispersion will increase with the propagation distance increasing. Thus some dispersion compensation fibers (DCFs) should be inserted [8] in order to decrease this accumulating dispersion.

Recently, because the structure design of the photonic crystal fiber (PCF) is flexible and dual-concentric-core fiber (DCCF) could attain high negative dispersion easily, some investigations of dual-concentric-core photonic crystal fiber (DCCPCF) [9-19] have attracted considerable attentions.

\section{Theoretical Approach and Design Structure}

The finite element method with perfectly matched absorbing layers boundary condition is used to investigate the guiding properties. Using the FEM, the PCF cross-section, with the finite number of air holes is divided into homogeneous subspaces where Maxwell's equations are solved by accounting for the adjacent subspaces. These subspaces are triangles that allow a good approximation of the circular structures. Using the PML, from Maxwell's curl equations the following vectorial equation is obtained [20], [21]

$$
\nabla \times\left([\mathrm{s}]^{-1} \nabla \times \mathrm{E}\right)-k_{0}^{2} \mathrm{n}_{\mathrm{eff}}^{2}[\mathrm{~s}] \mathrm{E}=0
$$

where $\mathrm{E}$ is the electric field vector, $k_{0}$ is the wave number in the vacuum, $\mathrm{n}_{\mathrm{eff}}$ is the refractive index of the domain, $[\mathrm{s}]$ is the PML matrix, $[\mathrm{s}]^{-1}$ is the inverse matrix of $[\mathrm{s}]$.

The chromatic dispersion D of PCFs is easily calculated from the $n_{\text {eff }}$ values vs. Wavelength using the following [22]

$$
\mathrm{D}=-\frac{\lambda}{\mathrm{c}} \frac{\mathrm{d}^{2} \operatorname{Re}\left[\mathrm{n}_{\mathrm{eff}}\right]}{\mathrm{d} \lambda^{2}}
$$

in $\mathrm{ps} /(\mathrm{nm} . \mathrm{km})$, where $\mathrm{Re}[$ neff] is the real part of effective refractive index neff , $\lambda$ is the wavelength, $\mathrm{c}$ is the velocity of light in vacuum. The material dispersion can be obtained from the three-term Sellmeier formula and is directly included in the calculation. In PCFs, the chromatic dispersion D is related to the additional design 
parameters like geometry of the air holes, pitch, and hole diameters. By optimizing these parameters, suitable guiding properties can be obtained.

To obtain a very high dispersion, lots of designs have been proposed, such as Wmodel, multi-cladding model, dual-concentric-core model [2-12] and so on. However, the dual-concentric-core model does better than the others. Based on this model, many works have been carried through to try to attain large negative dispersion. The first conventional DCCF with D of $-100 \mathrm{ps} /(\mathrm{km} . \mathrm{nm})$ around1.55 $\mu \mathrm{m}$ was proposed in document [9]. In [10-13], D were attained $-900 \mathrm{ps} /(\mathrm{km} . \mathrm{nm})$, $-1800 \mathrm{ps} /(\mathrm{km} . \mathrm{nm}),-5100 \mathrm{ps} /(\mathrm{km} . \mathrm{nm})$ respectively at $1.55 \mu \mathrm{m}$ by optimizing structural parameters. In recent years, along with the emergence of PCF, a novel type of DCCF based on PCF is appeared. It has a high index inner core and a defect ring of reduced holes diameter in the cladding. Researchers simulate and design this kind of fiber, and have attained very high negative dispersion [14-19]. However, such high refractive index doping is difficult to realize and high doping of the core is the increasing loss due to higher $\mathrm{GeO} 2$. Ni Yi et al. have proposed a novel dual-core DCF with D as large as $-18000 \mathrm{ps} /(\mathrm{km} . \mathrm{nm})$ [19]. Six small holes were introduced in the core of simple triangular PCF. However, it is difficult to fabricate the fiber exactly according to the design because it adopts a ring of much smaller holes and the accuracy of the structure parameter is difficult to control.

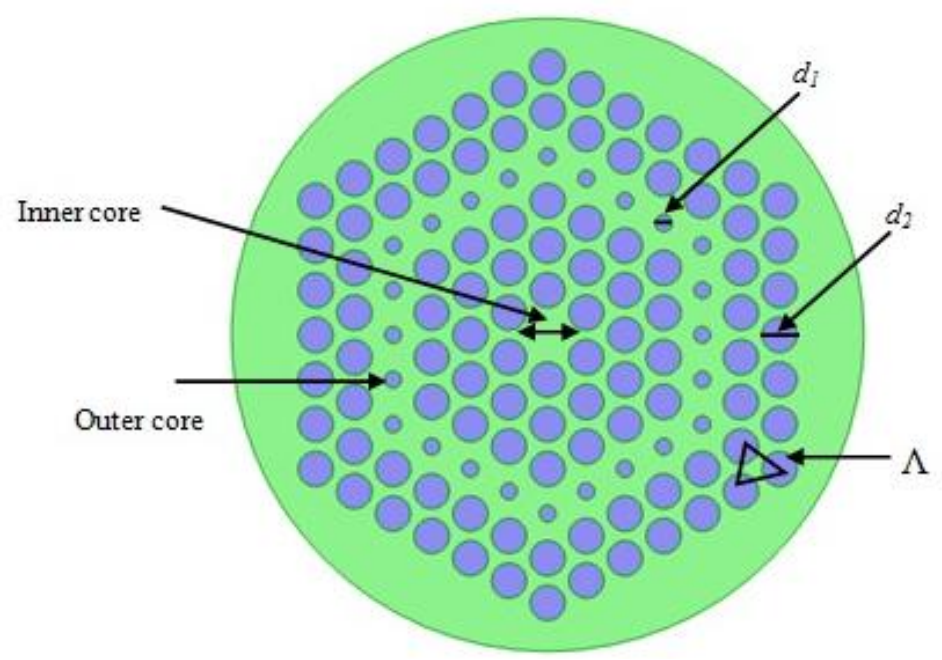

Fig. 1: The cross section of DCCPCF.

In this paper, FEM is used to investigate the chromatic dispersion and mode characteristics of DCCPCF. And detailed explanations about the propagation characteristics have been given. The cross section of DCCPCF is shown schematically in Fig. 1. It is obtained by introducing two kinds of holes with different diameters. The large hole's diameter is $d_{l}$, and the small hole's diameter 
is $d_{2}$. The pitch is $\Lambda$. The inner core is surrounded by the six large holes, and the outer core is consisted by the smaller holes.

\section{Numerical Results}

Due to the special structure of the DCCPCF, it determines there are at least two supermodes in the inner core and the outer core, respectively. One is fundamental mode, and the other is second mode. Typical field patterns in the two cores are shown in Fig. 2.

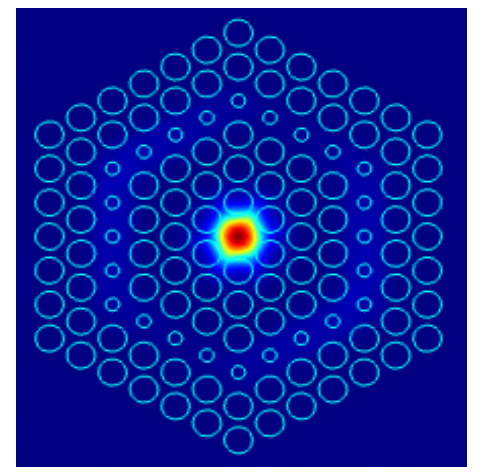

(a)

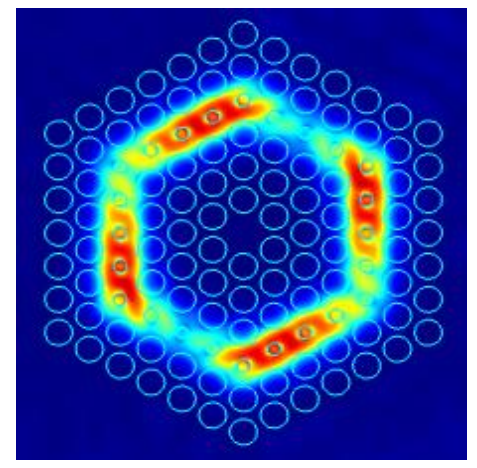

(b)

Fig. 2. Typical field patterns in these two cores; (a) In the inner core; (b) The outer core.

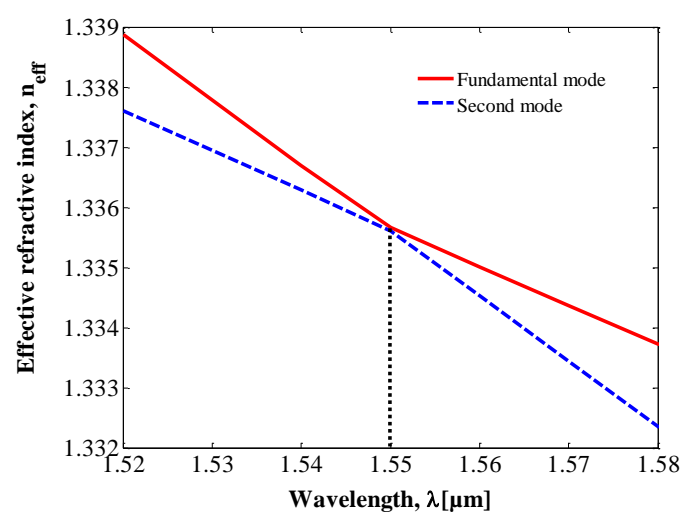

Fig. 3. Effective refractive indices versus the wavelength for the fundamental (solid line) and second-order (dashed line) modes. 
We first consider the DCF in Fig. 1 with lattice constant $\Lambda=1.27 \mu \mathrm{m}, d_{1}=0.48$ $\mu \mathrm{m}$, and $d_{2}=1.0 \mu \mathrm{m}$. By utilizing the FEM mode solver, we can obtain the effective refractive indices $\mathrm{n}_{\text {eff }}$ of the guided modes on the DCCPCF structure, shown in Fig. 3. For $\lambda<1.55 \mu \mathrm{m}$, the inner mode is the fundamental mode and the transmitted power is well confined in the central region. Around $\lambda=1.55 \mu \mathrm{m}$, the $\mathrm{n}_{\mathrm{eff}}$ of these two modes are so close and optical coupling takes place. Light field starts to spread out from the inner core to the outer core with a rapid change in the slope of the effective index curve, shown in Fig. 3. For $\lambda>1.55 \mu \mathrm{m}$, most power spreads from the inner core to the outer core and is well confined in the outer core region. Thus, the outer mode possesses higher $\mathrm{n}_{\mathrm{eff}}$ than the inner mode.

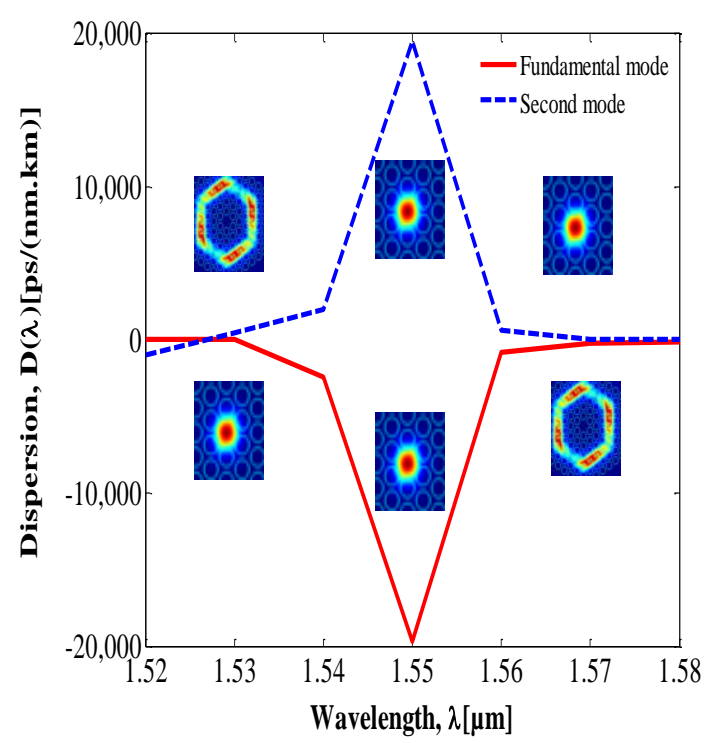

Fig. 4. The wavelength dependence of dispersion curves for optimum design parameter: $\Lambda=1.27 \mu \mathrm{m}, d_{1}=0.48 \mu \mathrm{m}$, and $d_{2}=1.0 \mu \mathrm{m}$, the insets showing the transverse field distributions at given wavelengths.

Base on above results, we can find that around $\lambda_{\mathrm{p}}=1.55 \mu \mathrm{m}$, due to optical coupling takes place between the fundamental mode and the second mode, the effective indices of these two modes have varied largely. It results in large D value at $\lambda p$, for both fundamental mode and second mode, as shown in Fig. 4. Fig. 4 shows the dispersion D curves of two leaky modes with peak value of \pm 20000 (ps/km.nm) around $1.55 \mu \mathrm{m}$. As presented by the insets of Fig. 4, for the inner core fundamental mode, the mode field mostly resides in the inner core region at shorter wavelength, gradually transfers from the inner core to the outer core as the wavelength increases, and comes into the outer core region at longer wavelength. For the outer core defect mode, a reverse transition trend is shown. 


\section{Conclusion}

Based on FEM method, a DCCPCF composed of pure silica and air is investigated thoroughly in this paper. Around $1.55 \mu \mathrm{m}$, it exhibits a negative chromatic dispersion as high as $-20000 \mathrm{ps} /(\mathrm{km} . \mathrm{nm})$. The chromatic dispersion and propagation characteristics of DCCPCF have been simulated systemically. The explanations to propagation states of the fundamental mode and the second mode are given elaborately.

\section{References}

[1] J. C. knight, "Photonic crystal fibers," Nature, vol. 424, pp. 847-851, 2003.

[2] J. C. Knight, T. A. Birks, P. S. J. Russell, and D. M. Atkin, “All-silica singlemode optical fiber with photonic crystal

cladding," Opt. Lett. vol. 21, pp. 1547-1549, 1996.

[3] M. D. Nielsen, C. Jacobsen, N. A. Mortensen, J. R. Folkenberg, and H.R. Simonsen, "Low-loss photonic crystal fibers

for transmission system and their dispersion properties," Opt. Express, vol. 12, pp. 1372-1376, 2004.

[4] K. Saitoh and M. Koshiba, "Highly nonlinear dispersion-flattened Photonic crystal fibers for supercontinuum generation in the Telecommunication window," Opt. Express, vol. 12, pp. 2027-2032, 2004.

[5] L. P. Shen, W. P. Hung, and S. S. Jian, "Design and optimization of photonic crystal fibers for broadband dispersion compensation, IEEE Photon. Technol. Lett., vol. 15, no. 4, pp. 540-542, Apr. 2003.

[6] V. Finazzi, T. M. Monro, and D. J. Richardson, "Small-core silica holey fibers: Nonlinearity and confinement loss trade offs," J. Opt.Soc. Amer. B, vol. 20, pp. 1427-1436, 2003.

[7] K. P. Hansen, "Dispersion flattened hybrid-core nonlinear photonic crystal fiber," Opt. Express, vol. 11, pp. 1503-1509, 2003.

[8] L.G. Nielsen, S.N. Knudsen, B. Edvold, T. Veng, D. Magnussen, C. C. Larsen, and H. Damsgaard, Optical Fiber Technology 6, 64 (2000).

[9] A.J. Antos and D. K. Smith, Lightwave Technol 12, 1739 (1994).

[10] J.L. Auguste, J. M. Blondy, J. Maury, J. Marcou, B. Dussardier, G. Monnom, R. Jindal, K. Thyagarajan, and B. P. pal, Optical Fiber Technology 8, 89 (2002).

[11] P.P. Bishnu and P. Kamna, Opt. Commun. 201, 335 (2002).

[12] J.L. Auguste, R. Jindal, and J. M. Blondy, Electron. Lett. 36, 1689 (2000).

[13] K. Thyagarajan, R. K. Varshney, P. Palai, A. K. Ghatak, and I. C. Goyal, IEEE Photon. Technol. Lett. 8, 1510 (1996).

[14] F. Gerome, J. L. Auguste, and J. M. Blondy, Opt. Lett. 29, 2725 (2004).

[15] F. Gerome, J. L. Auguste, and J. M. Blondy, Optical Fiber Communication Conference 2, 22 (2004). 
[16] A. Huttunen and P. Torma, Opt. Express 13, 627 (2005).

[17] T. Fujisawa, K. Saitoh, K. Wada, and M. Koshiba, Opt. Express 14, 893 (2006).

[18] S.G. Yang, Y. J. Zhang, X. Z. Peng, Y. Lu, S. Z. Xie, J. Y. Li, W. Chen, Z. W. Jiang, J. G. Peng, and H. Q. Li, Opt. Express 14, 3015 (2006).

[19] Y. Ni, L. Zhang Lei, L. An, J. D. Peng, and C. C. Fan, IEEE Photon. Technol. Lett. 16, 1516 (2004).

[20] K. Saitoh and M. Koshiba, "Full-vectorial imaginary-distance beam propagation method based on a finite element scheme: Application to photonic crystal fibers," IEEE J. Quantum Electron., vol. 38, no. 7, pp. 927933, Jul. 2002.

[21] S. Selleri, L. Vincetti, A. Cucinotta, and M. Zoboli, "Complex FEM modal solver of optical waveguides with PML boundary conditions," Opt. Quantum Electron., vol. 33, pp. 359-371, 2001.

[22] Begum F., Namihira Y., Razzak S.M.A., Zou N. Novel square photonic crystal fibers with ultra-flattened chromatic dispersion and low confinement losses. IEICE Trans Electron 2007:607. 